\title{
Indische Würmerkrankheiten*
}

\author{
Von R.F.G. MüLLER
}

$\mathrm{Zu}$ der hier gestellten Aufgabe ist vorweg zu bemerken, daß bei ihrer Untersuchung sich nicht in jeder Hinsicht Ergebnisse mit ausreichender Bestimmtheit erreichen lassen. Denn die indischen berufsärztlichen Lehren von den Würmerkrankheiten entwickelten sich aus volkstümlichen Heilungsbestrebungen, welche an sich in ihren Bewertungen schwierig zu überblicken sind und außerdem in ihrer Übernahme in die wissenschaftliche Heilkunde durch eine Zeitspanne getrennt sind, in welcher Textüberlieferungen fast gänzlich fehlen. Zu indogermanischen Begrenzungen hat schon KuHN in seiner Zeitschrift $^{1}$ grundlegend als «Segen gegen würmer » dargelegt, welche Hinweise aus dem asiatischen Osten angefügt werden könnten ${ }^{2}$. Aber ein derartig ausgedehnter Boden verleitet nicht weiter zu greifen, etwa bis nach Amerika, wie das zuweilen geschehen ist, anscheinend unter Nutzung durch «Ethnische Elementargedanken » ${ }^{3}$, die einen Gemeinplatz vermitteln können, welcher geschichtswissenschaftlich nicht unbedenklich ist. Dazu oder bei dieser Gelegenheit kommt in Frage, ob bei den ursprünglichen volkstümlichen Anschauungen der Inder von den Würmerkrankheiten der Begriff als «Vedische Zauber » ${ }^{4}$ oder «Aberglaube ${ }^{5}$ berechtigt ist, wie in den überliefernden Texten der Atharvaveda-Samhitā als «das Wissen von den Zaubersprüchen ${ }^{6}$ bekundet ist und allgemein beibehalten wurde. Wie der moderne Fachwissenschaftler die alten Anschauungen der Inder beurteilte, haben sie geschichtswissenschaftlich als belanglos zu gelten, sondern aus-

${ }^{*}$ Herrn Prof.Dr.med. Dr.phil. Dr.med.h.c. Dr.med.h.c. Paul Diepgen in Mainz zu seinem 85. Geburtstage, 24. November 1963.

${ }^{1}$ Z. vergl. Sprachforsch. (Berlin, bzw. Gütersloh) XIII (1864) 135-51.

2 Kleiweg De ZwaAn, Völkerkundliches und Geschichtliches über die Heilkunde der Chinesen und Japaner, S. 655: Würmer, Haarlem 1917; HüвоттеR, Die Chinesische Medizin, Leipzig 1929, S. 98.

${ }^{3}$ BASTIAN, Ethnische Elementargedanken in der Lehre vom Menschen, Berlin 1895.

${ }^{4}$ Hillebrandt, Ritual-Litteratur, Vedische Opfer und Zauber, S. 167, gibt zwar zu: «Die Abgrenzung von Glauben und Aberglauben kann nach meiner Meinung nur rein historisch oder gar nur individuell sein.» (Grundr. Indo-Arisch. Philol. Altertumskde. (Straßburg) III (1897) H. 2.

${ }^{5}$ Kreyenborg, Atharwaweda, warnte im Vorwort, S. XV, nachdrücklich (Hannover 1923).

${ }^{6}$ Winternitz, Geschichte der indischen Litteratur, Band I, Leipzig 1909, S. 103. 
schließlich wie diese selbst ihren Glauben «werteten». Die grundsätzliche Richtigstellung veranlaßt nachfolgend gelegentlich Bemerkungen, welche indologisch sonst unnötig wären.

Der Atharva-Veda vermittelt die ältesten Belege, welche die Würmerkrankheiten betreffen. Sie dürfen als volkstümlich beurteilt werden, weil die Sammlung dieser Lieder Belange einzelner Menschen betrifft, wobei kritisch auch solche der nicht-arischen, früheren Bevölkerung von Indien zuweilen nicht vollkommen ausgeschlossen werden können. Zum einführenden Überblick wird das Lied II, $31^{7}$ übertragen:

«(1.) Der große Mahlstein, welcher (des Gottes) Indra's ist, zerquetschend jeden Wurm, damit stampfe ich die Würmer zusammen, wie mit dem Mahlstein die Körner. (2.) Den gesehenen und den ungesehenen zerquetschte ich so dann; die Algaṇdu-s, die Śaluna-s, alle die Würmer lassen wir durch das Lied ${ }^{8}$ packen. (3.) Die Alganḍu-s töte ich mit der Mordwaffe; verbrannt, nicht-verbrannt, sie sind saftlos geworden; die übrig-gebliebenen und die nicht-übrig-gebliebenen überwinde ich durch das Lied; ebenso wie von den Würmern keiner übrig-bleiben soll. (4.) Den nach den Eingweiden strebenden, am Haupt und ferner an der Rippengegend befindlichen Wurm, den Avaskava, den Vyadhvara, die Würmer lassen wir durch das Lied packen. (5.) Die Würmer, welche im Gebirge, Walde, in den Kräutern, Viehherden und Gewässern sind, welche in unseren Leib eingedrungen sind, die gesamte Nachkommenschaft der Würmer töte ich.»

Die mannigfache Herkunft der Würmer und ihr Befall des menschlichen Körpers bekundet vielfältige Erfahrungen und damit auch alte Beobachtungen. Aber aus ihren Benennungen ist trotz mancher Versuche eine Kenntnis besonderer Formen nicht zu gewinnen; wie schon oft in den vedischen Texten richtet sich eine Aufmerksamkeit mehr auf die Wirkung und nicht auf eine Gestaltung, welche zuweilen nur angedeutet wird, wie etwa in dem folgenden Liede II, 32, welches nach dem Vorsatz 1 auf den Befall der Kuh zu beziehen wäre. In 2 wird als Gesamtansicht (viśvarüpa) des Wurmes vermerkt vier-äugig und färbig (sārañga, arjuna) [für be-

7 Atharva-Veda Samhitā, translated by Withney, edited by Lanman, Harvard Oriental Series, Vol. VII-VIII, Cambridge (Mass.) 1905, vermerkt bei allen Liedern frühere Bearbeitungen durch andere Autoren.

${ }^{8}$ Das Textwort vacas, wie auch in 4 , oder $v \bar{a} c$ in 3 ist nicht auf Zauber oder «spell » ${ }^{7} \mathrm{zu}$ beziehen, sondern [durch die wiederholten Hinweise] auf das hochbewertete «Heillied» [welches noch heut von Indern gesungen wird]; vgl. Nova Acta Leopoldina (Halle a.d. Saale) N.F. (1942) Nr. 74, S. 19. 
stimmte Farben unsicher]; in 6 werden bei ihm am Kopf zwei Hörner (śrnga) und ferner ein Giftbehälter (vișadhäna) angesagt. Das dritte Lied gegen die Würmer, V, 23, wendet sich vorzüglich gegen ihre Wirkung beim Knaben (kumära), welcher in 3 von ihnen an seinen Augen, Nasenlöchern und Zähnen befallen wird, mit in 7-8 mannigfach geschilderten Empfindungen. Hierbei werden doch Gestaltungen der Würmer erwähnt, wie in 4 ihre Ohrlöcher (karna), in 5 Unterarme (bāhu) oder 9 drei Köpfe und drei Höcker (kakud), ferner wiederholt [unsicher bestimmbare] Färbungen. Außer der tatsächlichen Bekämpfung der Würmer durch den singenden Priester bzw. Priesterarzt werden in II, 32 und noch mehr in V, 23 Götter und berühmte Verfasser vedischer Lieder zu Hilfe gerufen. Von letzten wird Agastya genannt, durch dessen bráhman die Würmer vernichtet werden sollen in II, 32, 3 und V, 23, 10. Die Bedeutung von brahman kommentiert zu erstem Beleg, allerdings spät (im 14.Jahrhundert n. Zw.), Säyaṇa ${ }^{9}$ als mantra-Lied für die Heilung, was einer grundsätzlichen Bewertung 8 immerhin entsprechen würde.

In XI, 9, 10 wird eine Schlange Arbudi angerufen, deren Biß befürchtet wird, im Anschluß an die Würmer, und in XII, 1, 46 wird nach einer beißenden Schlange (sarpa) ein Wurm erwähnt. Diese verbindende Annäherung erscheint nicht bedeutungslos für die Bewertung der Würmer. Anderseits mangeln besondere Behandlungsarten zur Bekämpfung der Würmer, welche möglicherweise erwartet werden könnten. Auf solche zielen die Texte im Kauśika-Sütra, die weit später anzusetzen sind, wenn auch eine genaue Datierung nicht möglich ist ${ }^{10}$, die kommentierenden Ergänzungen aus der ersten Hälfte des laufenden Jahrtausend stammen und Lehren der ärztlichen wissenschaftlichen der Inder bereits nutzen. Dabei sind entwicklungsgeschichtlich weitere Schwierigkeiten zu vermerken.

Die überlieferte Textfolge des Atharva-Veda ist nach grundsätzlichem Inhalt in der Anordnung im Kauśika-Sütra zusammengefaßt ${ }^{11}$, jedoch mit einem so kurzgefaßten Sütra-Stil, daß zu einem allgemeinen Verständnis Ergänzungen nicht umgangen werden können in Übersetzungsuntersuchungen ${ }^{12}$, welche zuweilen nicht unbedenklich erscheinen. Die Darlegung

${ }^{9}$ Kurze Lebensbeschreibung durch KLemm, Das Șaḍviṃ̧̧abrāhmaṇa, Diss. Leipzig (Gütersloh 1894), S. 15-9.

${ }^{10} \mathrm{Vgl}$. Introduction zur Textausgabe von Bloomfield, The Kauçika-Sütra, New Haven 1890.

11 Acta Hist. Sci. Nat. Med. (Kopenhagen) VIII (1951) $10 \mathrm{ff}$.

12 Caland, Altindisches Zauberritual, Amsterdam 1900; vgl. auch Z. dtsch. morgenl. Ges. 53 (1899) 223. 
solcher Einzelheiten würden hier zu weit führen. In Zusammenfassung ist die vedische Wertung des Heil-Liedes im Atharva-Veda auch im KauśikaSütra 27, 14-20 und 29, 20-26 bewahrt, wo das Absingen der Lieder II, 31 und V, 23 vorausgesetzt wird. Auch vedische Heilmaßnahmen wurden eingehalten, die oft in einer äußeren Behandlung bestanden ${ }^{13}$, welche von den Priesterärzten ziemlich bevorzugt wurden. Denn bei den arischen Einwanderungen nach Indien war ein Genuß von Nähr- und Heilpflanzen sehr von Erfahrungen der schon ansässigen Bevölkerungen abhängig, ohne daß deren Einfluß überschätżt werden darf ${ }^{14}$. Jedenfalls ist im Kauśika-Sütra eine ausgedehnte Nutzung vom Genuß der Heilpflanzen bereits erkennbar, auch im Kampf gegen die Würmer.

$\mathrm{Zu}$ den bisherigen Belegen ist noch die Liedersammlung vom Rg-Veda zu berücksichtigen, welche insgesamt als die älteste gilt. Jedoch das Lied I, 191 wird von den Fachgelehrten allgemein als ein später Nachtrag beurteilt, der auf die Abwehr der Würmer bezogen werden kann. Denn der übliche Ausdruck $k r m i$ für Wurm wird hierbei (wie auch im ganzen $R g$-Veda) nicht gebraucht, und nur inhaltlich ist ein entsprechender Bezug annehmbar, wie etwa wegen der Bezeichnung in 8 durch «gesehene und ungesehene» [Würmer]. Auch die Eigennamen sind ungesichert, wie in 1: Kankkata und Prakañkata in 7 (von Sãyaṇa spät als "Schlange» kommentiert) oder in 15: Kușumbhaka, ein kleines Wesen, das mit dem Stein erschlagen und bei dem sein Gift (wie auch sonst öfter) hervorgehoben wird. Letztes wird in 16 auch auf einen "Skorpion» bezogen; in 7 werden "Stechmücken» angenommen. Diese Erklärungen deuten, wie schon im Atharva-Veda, auf einen tierkundlich erweiterten Begriff der arischen Wertung der Würmer.

Ein ausreichender Rückblick auf die Entwicklung der beruflichen und wissenschaftlichen Sammelwerke der ärztlichen Lehrer in Indien ist nur bedingt möglich wegen mangelnder Überlieferungen aus der Zwischenzeit. Gemäß den Eigenangaben nutzten die großen Sammelwerke die acht Glieder oder Traktate des Wissens vom Leben, äyurveda, dessen Bezeichnung deswegen geschichtskritisch als «Altmedizin » anzusprechen ist, wenn auch die späteren Inder diesen Ausdruck für jede, ihnen bodenständig erscheinende Medizin beibehielten. Nach grober Schätzung faßte um die Mitte des ersten Jahrtausends n.Z. Vägbhața in den nach ihm benannten Sammlungen als Grundlage die alten Heilbestrebungen für den Gesamtleib oder Rumpf

13 Nova Acta Leopoldina, N.F., Nr. 1958, S. $13 \mathrm{ff}$.

14 Wien. Z. Kde. Süd-u. Ostasiens (Wien) IV (1960) 14ff. 
(kāya) zusammen, während der sagenhafte berühmte Arzt Suśruta für die Wundärzte ihr einschlägiges Fachbereich (śalya) in den Vordergrund stellte. Die jeweils übrigen der acht Traktate, deren Texte nicht unmittelbar erhalten geblieben sind, wurden ursprünglich dem Nachtrag $\left(u t^{\circ}\right)$ der Sammlungen angefügt ${ }^{15}$. Die grundsätzliche Richtung der Glieder der «Altmedizin» muß auf Heilbestrebungen gezielt haben, denen kurz Krankheitsanzeichen einleitend vorgesetzt sein dürften. Wenn aber, wie in den $V$ ägbhata-Schulen, dieser Heilungsabsichten $\left(c i^{\circ}\right)$ in eigenen Lehrabschnitten von den Krankheitserklärungen ( $\left.n i^{\circ}\right)$ getrennt wurden, so muß eine solche Anordnung als nachträglich beurteilt werden, zumal die Texte der Sammelwerke bis zum Beginn des jetzigen Jahrtausends manche Über- und Umarbeitung erfuhren, wie aus den Kommentaren erkennbar ist. Letzte zeigen am wenigsten die wundärztlichen Lehren in Suśruta-Samhitä $u t^{\circ}$, in deren regelmäßigen Abschnittseinleitungen von einer Abwehr (pratiședha) der Krankheiten gesprochen wird, also mit einer Ausdrucksweise, welche an die vedische Wertung der Krankheiten als unholde Erzeuger der Leiden erinnern kann, so auch in $u t^{\circ} 54$, wo die Abwehr der Würmer gelehrt wird. Allerdings ist auch hier eine Textumarbeitung schon in der Einleitung 3-5 wegen der Ausdrucksweise des späteren Textsatzbaues erkennbar und ferner durch den Zusatz der Galle (pitta) als Krankheitsursache. Denn ganz allgemein gelten drei Fehler (doșa), so in allen Krankheits- und Heilungslehren ${ }^{16}$. Es ist daher begründet, daß die Einfügung von der Galle hier die regelmäßige Aufzählung mit Wind und Schleim ergänzen soll, während die Lehren von den Würmern weiterhin ursächlich nach den alten Wertungen bewahrt blieben.

Suśruta-Sam̧itā $u t^{\circ} 54$ wird übertragen: «(1.) Nunmehr werden wır alsdann den Lehrabschnitt von der Abwehr der Würmerkrankheit auseinandersetzen, (2.) wie der heilzuteilende ${ }^{17}$ (Götterarzt) Dhanvantari ansagte: (3.) Durch unverdaute, für Nahrungsaufnahme nicht günstige, unverträgliche und schmutzige Speisen, die ohne Anstrengungen, bei Tagesschlaf, schwer, allzu glitschig und kalt gelten, (4.) durch Bohnenmehl, genossenes Backwerk, in Verbindung mit Stengeln und Wurzeln vom Lotos, durch Blätter, Gemüse, Gärtrank, sauren Milchsaft und gekochten Zuckerrohrsaft, (5.) durch Sesamkörnerbrei, Fleisch von Sumpflandtieren,

15 Centaurus (Kopenhagen) I (1950/51) 97-116.

${ }^{16}$ Jb. Museum Völkerkde. Leipzig (Berlin) XVII (1960) 76-93.

${ }^{17}$ Vgl. Mitt. Inst. Orientforsch. (Berlin) VIII (1961) 120 : bhagavant. 
Ölkuchen und durch süß oder sauer laufende Getränke wallt der Schleim und die Galle (pitia) auf. (6.) Die Würmer bewirken unter den vielfach wirkenden und mannigfach anhaftenden in der Lagerung der rohen und gekochten ${ }^{18}$, ferner bei den Kotbildungen der Schleim und durch die beiden Gefäße, welche Blut erzeugen, einen meist vermerkten Antrieb.

(7.) Bei zwanzig der Würmerbildungen ist eine mannigfache Entstehung vermerkt als Kot, Schleim und Blut, deren (Bildungen) wir ausführlich ansagen werden. (8.) Ajava, Vijava, Kipya, Cipya, Gaṇdūpada, Cāru und Dvimukha ${ }^{19}$ sind zu erkennen als aus Kot entstanden. (8.) Sie sind hell, dünn, aber beißend und eilen im Aftergang weiter, und von ihnen später durch Schweife ausgebreitet. (10.) Stiche, Schwäche vom Feuer (der Verdauung), Bleichheit, Verstopfung, Kräfteschwund, Ekel, Eßunlust, Herzleiden ${ }^{20}$, Durchfall entstehen durch die aus Kot erzeugten (Würmer). (11.) Rot sind die Gandüpada-s, lang, im Aftergang sich mit Beißen niederlassend, mit Stichen blähend, Stuhlgang lösend und das Kochen der Verdauung schwinden machend. (12.) Darbhapușpa, Mahāpușpa, Praluna, Cipița, Pipilika ${ }^{21}$ und Däruṇa sind aus dem Kot gewordene (Würmer). (13.) Am Körper behaart, am Kopf behaart, am Schweif, dunkel, gerundet, wie Korn gewachsen, Sprossen bewirkend, licht und schlank. (14.) Mark essend, Augen anbohrend, Gaumen und Ohren als Schlange, am Haupt und Herzkrankheiten, Erbrechen und Erkältung sind sie bewirkend. (15.) Keśa Roma $^{\circ}$, Nakhâda, Dantâsa, Kikkiśa, Kușthaja und Saparisarpa ${ }^{22}$ sind zu erkennen als aus Blut entstanden. (16.) Sie sind sowohl rot wie schwarz, sowohl glatt wie üppig; sie werden am Standort des Blutes erzeugt, meist als schlechte Ausfertigungen.»

${ }^{18} \mathrm{Zu}$ «der rohen und gekochten» ist Speise zu ergänzen; eine Kenntnis vom Organ des Magens und des betreffenden Darmes ist bei den altindischen Ärzten nicht nachweisbar, auch nicht in den späteren tibetischen Abbildungen; vgl. Sudhoffs Arch. (Leipzig) 28, (1935) 242, Abb. 4, und S. $259 \mathrm{f}$.

${ }^{19}$ Dvi-mukha $=$ zwei-mündig ist in der Ausdrucksweise verständlich. Sonst sind die Namen (wie schon in den vedischen Texten) aus ihrer Wortbildung kaum sicherbar, werden auch dahin in der Regel nicht kommentiert, so daß nur die folgenden Schilderungen etwas nutzbar sind.

${ }^{20}$ Suśruta-Samhitā $u t^{\circ} 43$ lehrt bei der Herzkrankheit auch eingehend die Ursache durch Würmer; Pagine di Storia della Medicina, A. (Roma) IV (1960) N. 3.

${ }^{21}$ Pipilika ist eine gebräuchliche Benennung der Ameise und so bestimmbar.

22 Die Namen sind hier verständlicher, wie Haupthaar-, Körperhaar-, Nägel-Fresser, ZahnFresser; $j a=$ entstanden (wie auch früher gebraucht) bei «aus ,Aussatz' entstanden» oder -sarpa $=$ kriechend, Schlange. 
Ab 17 werden inhaltlich Angaben wiederholt, welche ursprünglich als Zusammenfassung aufgefaßt werden können, obwohl sie durch den Nachträglichen Textvorsatz $3 \mathrm{ff}$. als Wiederholung erscheinen. Nach einem kurzen Hinweis auf die Heilbarkeit der Wurmerkrankung ist der umfangreiche Textschluß bis 40 den eigentlichen Heilungsmaßnahmen gewidmet. Dabei ist zu beachten, daß eine Behandlung durch unmittelbare Vernichtung der Würmer, wie sie in den vedischen Texten sehr betont wurde, mangelt. Da die Wundärzte durchaus erfahren waren in solchen Maßnahmen, ist kritisch zu folgern, daß das grundsätzliche Zerquetschen der Würmer schon in den Lehren der «Altmedizin» in den Hintergrund getreten war gegenüber dem zunehmenden Genuß von vorzüglich pflanzlichen Heilmitteln. Letzte werden in Zusammenhang mit Verdauungsmaßnahmen auch von den Wundärzten in reichlichem Umfange übernommen, woraus eine Gesamtumstellung in den Sammelwerken erkennbar ist. Die Bestimmung der Heilpflanzen in den alten Texten ist schwierig, weil sie in den wissenschaftlichen Fachwerken regelmäßig auf modernen botanischen Einschätzungen beruhen ${ }^{23}$. In den Kommentaren tauchen oft Änderungen auf, wobei nicht feststellbar ist, ob es sich um Ersatznamen oder Ersatzpflanzen handelt. Sogar in einem Lehrtext vom Soma, dem seit den Veden berühmten Heiltrank, führt Suśruta-Samhitā $c i^{\circ} 29$ zwei Dutzend solcher fraglicher Bezeichnungen an ${ }^{24}$. So entstand bei den neuzeitlichen Indern der Glaube an eine «synonyme» Heilpflanzenbenennung ${ }^{25}$.

Die Lehre von den Würmerkrankheiten bringen auch die VägbhațaSchulen, im $H_{r}$ daya $n i^{\circ}$ 14, 42-56 und ihre Heilungsbestrebungen $c i^{\circ} 20$, $19-35^{26}$ und im Samgraha $n i^{\circ} 14$ sowie $c i^{\circ} 22$. In beiden Texten sind jene von den Würmern solchen von Hautkrankheiten angefügt, was für die Anordnung in der «Altmedizin» nicht vorausgesetzt werden kann und eine spätere Entwicklung bekundet. Auch die Benennungen der Würmer weisen Änderungen auf, wie auch die Ausgestaltung der Heilpflanzen. Hingegen

${ }^{23}$ U.Ch. Dutr, The Materia Medica of the Hindus, Kalkutta 1922, der noch am meisten geschichtlich Rücksicht nimmt, geht in der Regel seine Textbelege nur bis zum 11.Jh. n. Zw. auf Cakrapānidatta zurück.

${ }^{24}$ Asiatica, Festschrift Friedrich Weller, Leipzig 1954, S. 428-41.

${ }^{25}$ The Caraka Samhitā, ed. by Gulabkunverba Ayurvedic Society (Jamnagar 1949) bringt in Vol. VI, S. 1-28, ein umfangreiches Verzeichnis von Heilpflanzen, in welchen oft über fünfzig «Synonyms» vermerkt sind.

26 Übersetzung von Hilgenberg und Kirfel, Vägbhața's Aștāingakṛdayasamhitā, Leiden 1941. 
ist die Herkunft aus Schleim, Blut und Kot die gleiche und ebenso die Zahl ihrer Arten. Die Hrdaya-Texte blieben gültig oder wurden wieder belebt in der Neuzeit (nach der Mitte des 19. Jahrhunderts) durch Paraneśvara in seinem $H_{r}$ dayapriya $16,41 \mathrm{ff}$.

Wenn auch eine Beachtung der Altmedizin gegenüber jener der großen Sammelwerke zurücktrat, so wurde sie doch noch weiterhin berücksichtigt. Das bekundet die Cikitsākalikā 280 des Tissaṭa, der frühesten im 11.Jahrhundert ${ }^{27}$ auch auf die Würmer hinwies. Derartige Verhältnisse können dazu beigetragen haben, daß in der Caraka-Schule, deren Einstellung oft Erkenntnis-grundsätzlich ausgerichtet ist, die altmedizinische Heilkunde erst spät durch den Überarbeiter und Ergänzer der Texte Dṛ̣̂abala etwa im 9. Jahrhundert aufgenommen wurde. In Caraka-Samhitā sü $\bar{u}^{\circ} 19$ werden die zwanzig Arten der Würmer mit ihren Einteilungen der Entstehung aus Blut, Schleim und Kot angekündigt. Ihre ausführliche Schilderung bringt aber erst $v i^{\circ} 7,9 \mathrm{ff}$. Dabei werden aber ihre zwanzig Arten durch Zufügung ihrer Entstehung aus Schmutzstoffen (mala) zu jener aus Kot, Schleim und Blut etwas erweitert. Ohne auf eine zahlenmäßige Beurteilung einzugehen, auf andere Besonderheiten (auch in anderen Lehrtexten) oder Änderungen oder Austausch der Benennungen, ist doch die große beachtliche Anteilnahme der indischen Ärzte aus diesen Vorgängen zu erkennen.

Noch im 16. Jahrhundert wurde in dem bekannten Buch Bhävaprakāśa (II, 7) an der Lehre von den zwanzig Wurmarten aus Schleim, Blut und Kot festgehalten und ebenso im Ayurveda Vijnanam durch den modernen Hinduarzt B. L. Sen (Vol. II, p. 129-132, Kalkutta 1916), obwohl dieser gelegentlich die jetzige europäisch-amerikanische Wissenschaft nutzte. Bei einem Gesamtrückblick auf die Entwicklung indischer Medizin ergibt sich demnach, daß in ihr seit der Altzeit zwanzig Arten von Würmern bewahrt blieben, die dem Schleim, Blut und Kot entstammten, obwohl sonst in der Krankheitslehre als Ursachen nur Wind, Galle und Schleim galten. Diese Sonderung der Wurmwertung bekundete somit ihre gesteigerte Beachtung und auch etwas Formanschauung. In einer Abbildung ${ }^{28}$ ist ein aus dem linken Ohrloch der bösen Göttin Śridevi kriechender Wurm zu erkennen, der sonst als Schlange gilt, und solche Schlangen wurden schon im Rgveda sogar als «Drachen » ${ }^{29}$ angesprochen. Auch diese Belege bekunden die Er-

27 Jolly, Z. disch. morgenl. Ges. 40 (1906) 468.

28 Mitt. Museum Völkerkde. Hamburg 1917, Tafel 2, Nr. 11, Abb. 16.

${ }^{29}$ Harvard Oriental Series (Cambridge, Mass.) 36 (1957) 153. 
weiterung des indisch-ursprünglichen Begriffes vom Wurm. Trotz der Selbständigkeit der indischen Medizin und den daraus begründeten Bedenken gegen Gemeinplätze sind aber doch Einwirkungen allgemeiner Denkweisen gerade beim «Wurm » oder «Parasiten» nicht ganz auszuschließen, welche auch seine mannigfache indischen Bewertungen miterklären könnten. Denn Diepgen ${ }^{30}$ erwies, daß seit alter Vergangenheit und gesteigert gegen die Neuzeit unter verschiedenen Voraussetzungen und Folgerungen entsprechende Beurteilungen solcher Gebilde erfolgten.

${ }^{30}$ DiePgen, Geschichte der Medizin, Band I-II, 1 und 2, vgl. Sachregister, Berlin 1940-1955. 